\title{
Single-Incision Laparoscopic Splenectomy and Splenic Autotransplantation for an Enlarged Wandering Spleen with Torsion
}

\author{
Shunsaku Katsura ${ }^{1}$ Daichi Kawamura ${ }^{2}$ Eijiro Harada ${ }^{2}$ Tadahiko Enoki ${ }^{2}$ Kimikazu Hamano ${ }^{2}$ \\ ${ }^{1}$ Division of Pediatric Surgery, Department of Surgery and Clinical \\ Science, Yamaguchi University Graduate School of medicine, Ube, \\ Yamaguchi, Japan \\ 2 Department of Surgery and Clinical Science, Yamaguchi University \\ Graduate School of medicine, Ube, Yamaguchi, Japan \\ Address for correspondence Shunsaku Katsura, MD, Division of \\ Pediatric Surgery, Department of Surgery and Clinical Science, \\ Yamaguchi University Graduate School of medicine, 1-1-1 \\ Minamikogushi, Ube, Yamaguchi 755-8505, Japan \\ (e-mail: shunsaku@yamaguchi-u.ac.jp; shunsaku427@yahoo.co.jp).
}

Eur J Pediatr Surg Rep

\begin{abstract}
Keywords

- wandering spleen

- single umbilical incision

- autotransplantation

A wandering spleen is a rare condition in which the spleen is not located in the left upper quadrant, but instead is found in the lower abdomen or in the pelvic region because of the laxity of the peritoneal attachments. The unusually long pedicle is susceptible to twisting, which can lead to ischemia, and eventually to necrosis. We herein report a case of an enlarged wandering spleen with torsion, successfully treated by single-incision laparoscopic splenectomy and autotransplantation. The transplanted splenic tissues could be identified on a spleen scintigram obtained 3 months after the surgery. HowellJolly bodies were not observed in blood specimens. This procedure is able to prevent an overwhelming postsplenectomy infection, and leads to satisfactory cosmetic results.
\end{abstract}

\section{Introduction}

A wandering spleen is a rare condition in which the spleen is not located in the left upper quadrant, but instead is found in the lower abdomen or in the pelvic region because of the laxity of the peritoneal attachments. ${ }^{1}$ The unusually long pedicle is susceptible to twisting, which can lead to ischemia, and eventually to necrosis. The clinical presentation of patients with this condition may be acute abdominal pain secondary to splenic torsion or intestinal obstruction, or an abdominal mass caused by splenic congestion and capsular tension. 2,3 We herein report the successful laparoscopic management of an enlarged wandering spleen with torsion. We performed single-incision laparoscopic splenectomy and autotransplantation because the fixation of the enlarged spleen seemed to be difficult to perform under a laparoscopic procedure. This procedure was useful for preventing an overwhelming postsplenectomy infection, and led to satisfactory cosmetic results.

\section{Case Report}

A 13-year-old female was hospitalized for intermittent abdominal pain and a lower abdominal mass. On physical examination, we palpated a slightly tender, mobile mass in the left side of the lower abdomen. The laboratory results were within the normal ranges. On admission, computed tomographic scan showed an enlarged spleen $(168 \times 86 \times 53 \mathrm{~mm})$ on the left side of the abdomen (-Fig. 1a) and a "whirl sign" of the splenic vein (-Fig. 1b). Splenic ischemia was not observed. On the basis of the diagnosis of an enlarged wandering spleen with torsion, we performed single-incision laparoscopic splenectomy and autotransplantation. The single-incision laparoscopic splenectomy was performed through a 3-cm vertical umbilical incision. An EZ Access (Hakko, Tokyo, Japan) was placed in position through the incision. Through this EZ Access, three ports (EZ Trocar $5 \mathrm{~mm}$, Hakko) were introduced. The peritoneal space was inflated with carbon dioxide gas, and a flexible received

June 12, 2013

accepted after revision

August 16, 2013
DOI http://dx.doi.org/

10.1055/s-0033-1357262. ISSN 2194-7619. (c) Georg Thieme Verlag KG

Stuttgart · New York
License terms

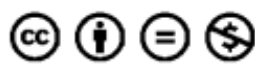




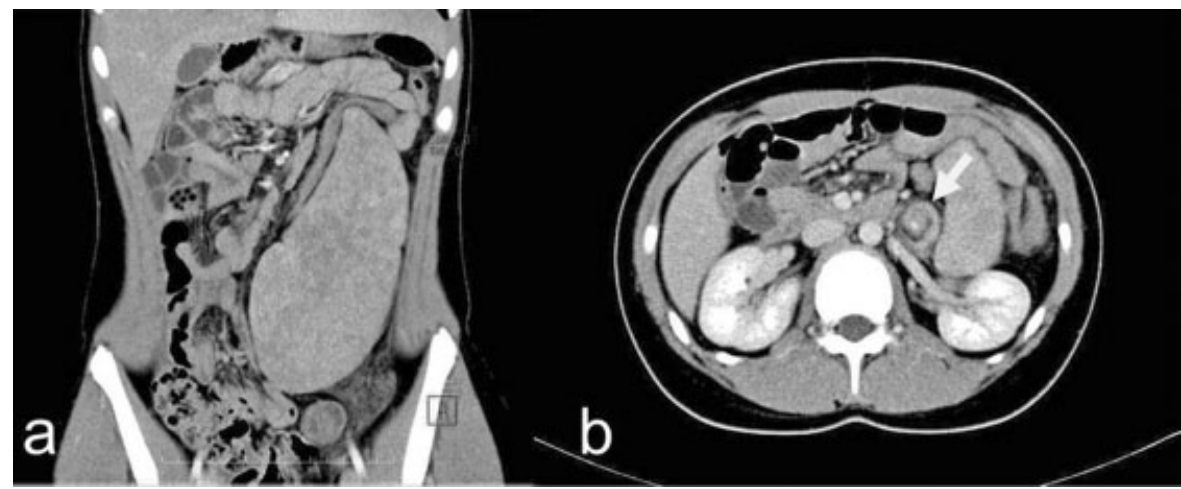

Fig. 1 (a, b) A computed tomographic scan showed an enlarged spleen $(168 \times 86 \times 53 \mathrm{~mm})$ in the left side of the abdomen, and a "whirl sign" of the splenic vein (arrow).

camera was inserted through the 5-mm port. The spleen was gently lifted up using a Mini Loop Retractor II (Covidien, Tokyo, Japan) inserted from below the costal margin on the left clavicular line and a polyurethane sponge (Securea) (HOGY, Tokyo, Japan) for an endoscopic surgical spacer. The spleen was congested, enlarged and wandering because of the absence of spleen-fixing gastrosplenic or splenorenal ligaments. The splenic pedicle was twisted 360 degrees in a counterclockwise rotation. The splenic artery and vein were transected with an endoscopic stapler. The excised spleen was placed in a retrieval bag and morcellated with forceps. The excised spleen weighed $176 \mathrm{~g}$. We transplanted six pieces of morcellated splenic tissue, weighing $50 \mathrm{~g}$ total, into a pouch created in the gastrocolic omentum through the single umbilical incision (-Fig. 2). The postoperative course was uneventful. The patient was discharged home on postoperative day 6 , and received a pneumococcal vaccination. The transplanted splenic tissues could be identified on a spleen scintigram obtained 3 months after the surgery ( - Fig. 3a, b).

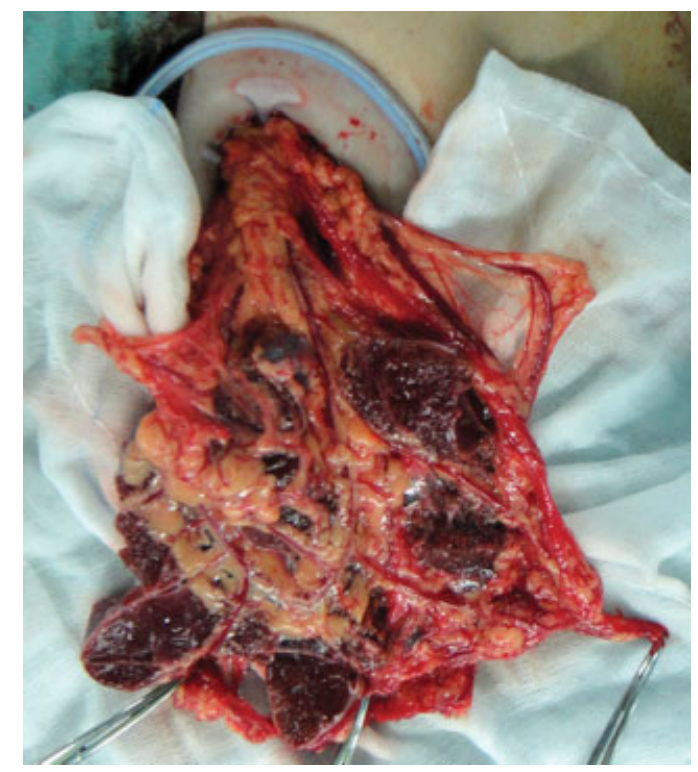

Fig. 2 Splenic tissues were transplanted into pouches created in the omentum through the single umbilical incision.
Howell-Jolly bodies were not observed in blood specimens. The patient has been in good health for a year after the operation.

\section{Discussion}

In the past, a splenectomy was the standard recommended treatment for a wandering spleen, independent of the presence or absence of torsion. ${ }^{1,3}$ However, the increasing knowledge of the spleen's role in the reticuloendothelial system makes the unnecessary removal of the spleen unwarranted. It is recommended that splenectomy should be avoided to prevent the increased risk of an overwhelming postsplenectomy infection, especially in children. Thus, splenopexy is recommended for preventing the complications associated with splenectomy, while preserving the splenic function, when there are no signs of splenic infarction. ${ }^{4}$

Several types of splenopexy techniques have been presented: simple sutures between the capsule and diaphragm, placement of the spleen in a posterolateral extraperitoneal pocket, and sutures made in the absorbable mesh-enwrapped spleen. ${ }^{5-8}$ When the spleen is highly enlarged, splenopexy may be difficult. The solution is to perform an elective partial splenectomy and fix the residual hemispleen to the diaphragm, or to perform splenectomy and splenic autotransplantation. Both procedures have been reported when laparotomy was performed, but there have been no reports of these treatments being performed laparoscopically. ${ }^{9,10}$ It is very difficult to perform an elective partial splenectomy and fix the residual hemispleen to the diaphragm during laparoscopic procedure. On the other hand, we thought that it would be possible to perform splenectomy and splenic autotransplantation laparoscopically. With respect to splenectomy, several case reports have been published on both the feasibility of single-incision laparoscopic splenectomy and the favorable cosmetic outcomes. ${ }^{11,12}$ After the spleen is laparoscopically removed, it is placed in a retrieval bag and morcellated with forceps. Splenic autotransplantation is able to be done by transplanting part of the splenic morcellated tissue into pouches created in the omentum through the single umbilical incision.

Splenic autotransplantation is a method of preserving the organ function that is performed mainly for trauma patients 


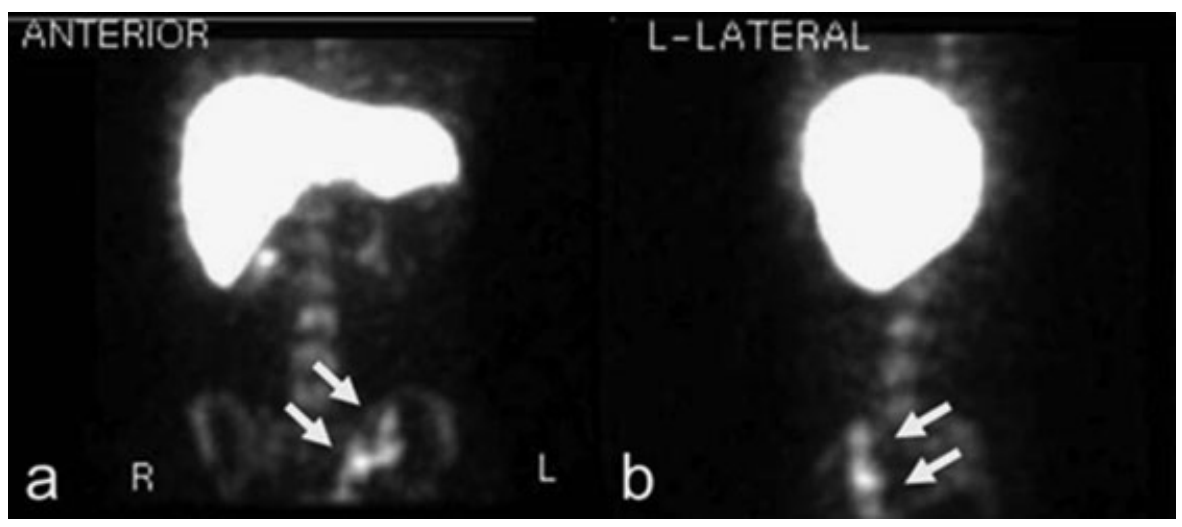

Fig. 3 (a, b) Splenic scintigraphy performed 3 months after surgery showed identifiable transplanted splenic tissues (arrow).

whose injured spleen must be resected. Multiple human and animal studies have established that splenic autotransplantation is a relatively safe and easily performed procedure. ${ }^{13}$ Iinuma et al reported a $100 \%$ success rate for splenic autotransplantation into the omental pouch in rats. ${ }^{14}$ The amount of splenic tissue necessary to prevent an overwhelming postsplenectomy infection in humans, and the optimal graft size, have not been established. Variable amounts of splenic tissue, ranging from 24 to $100 \mathrm{~g}$, have been transplanted with successful regeneration. ${ }^{9}$ In the present case, we transplanted six pieces of splenic tissue, weighing a total of $50 \mathrm{~g}$. Because torsion of autotransplanted splenic tissue has been reported as a rare complication, careful observations will be necessary in this case. ${ }^{15}$

The assessment of splenic function after autotransplantation in humans must rely on indirect laboratory and radionuclide scanning techniques. The presence of Howell-Jolly bodies on peripheral blood smears is an accepted indication of the absence or insufficiency of splenic filtration function. ${ }^{13}$ Scintigraphy has universally demonstrated functional splenic tissue with the uptake of the radionuclide. However, the mere presence of splenic tissue does not necessarily imply normal immune function. ${ }^{9,13}$

In the present case, the spleen showed enlargement and the torsion of its pedicle, but no infarction. Therefore, we selected single-incision laparoscopic splenectomy and autotransplantation to solve both the problems related the risk of infection after splenectomy and the cosmetic problems, and obtained a satisfactory outcome for the patient. No Howell-Jolly bodies were observed in blood specimens, and the transplanted splenic tissues could be identified on a spleen scintigram obtained 3 months after surgery. However, it remains unclear whether the regenerating tissues will prevent severe infections or reduce the incidence of morbidity or mortality caused by overwhelming postsplenectomy infection. Therefore, meticulous observation of this patient will be necessary.

\section{References}

1 Magowska A. Wandering spleen: a medical enigma, its natural history and rationalization. World J Surg 2013;37(3):545-550
2 Soleimani M, Mehrabi A, Kashfi A, Fonouni H, Büchler MW, Kraus TW. Surgical treatment of patients with wandering spleen: report of six cases with a review of the literature. Surg Today 2007;37(3): 261-269

3 Steinberg R, Karmazyn B, Dlugy E, et al. Clinical presentation of wandering spleen. J Pediatr Surg 2002;37(10):E30

4 Fiquet-Francois C, Belouadah M, Ludot $\mathrm{H}$, et al. Wandering spleen in children: multicenter retrospective study. J Pediatr Surg 2010; 45(7):1519-1524

5 Fukuzawa H, Urushihara N, Ogura K, et al. Laparoscopic splenopexy for wandering spleen: extraperitoneal pocket splenopexy. Pediatr Surg Int 2006;22(11):931-934

6 Martínez-Ferro M, Elmo G, Laje P. Laparoscopic pocket splenopexy for wandering spleen: a case report. J Pediatr Surg 2005;40(5): 882-884

7 Schaarschmidt K, Lempe M, Kolberg-Schwerdt A, Schlesinger F, Hayek I, Jaeschke U. The technique of laparoscopic retroperitoneal splenopexy for symptomatic wandering spleen in childhood. J Pediatr Surg 2005;40(3):575-577

8 Hirose R, Kitano S, Bando T, et al. Laparoscopic splenopexy for pediatric wandering spleen. J Pediatr Surg 1998;33(10): 1571-1573

9 Takayasu H, Ishimaru Y, Tahara K, Otani Y, Yamagishi J, Ikeda H. Splenic autotransplantation for a congested and enlarged wandering spleen with torsion: report of a case. Surg Today 2006;36(12): 1094-1097

10 Esposito C, Settimi A, Centonze A, Damiano R, Maglio P, Esposito G. Enlarged wandering spleen treated with hemisplenectomy and fixation of the residual spleen. Pediatr Surg Int 2005;21(6): 488-490

11 Bell R, Boswell T, Hui T, Su W. Single-incision laparoscopic splenectomy in children. J Pediatr Surg 2012;47(5):898-903

12 Oyama K, Sasaki A, Chiba T, Nitta H, Otsuka K, Wakabayashi G. Single-incision laparoscopic splenectomy for idiopathic thrombocytopenic purpura: report of a case. Surg Today 2011;41(8): 1091-1094

13 Pisters PWT, Pachter HL. Autologous splenic transplantation for splenic trauma. Ann Surg 1994;219(3):225-235

14 Iinuma H, Okinaga K, Sato S, Tomioka M, Matsumoto K. Optimal site and amount of splenic tissue for autotransplantation. J Surg Res 1992;53(2):109-116

15 Ando K, Miyano T, Yamataka A, et al. Torsion of autotransplanted splenic tissue in Gaucher disease. J Pediatr Surg 1997;32(5): 747-749 